Research Paper

\title{
Pretreatment biomarkers as prognostic predictors of survival in patients with Pancreatic Cancer treated with Gemcitabine- based Therapy and 5-Fluorouracil: Neutrophil-to-lymphocyte ratio vs Platelet-to-lymphocyte ratio
}

\author{
Yungu Chen"\#, Yuan Liao"\#, Lek Man Lam³, Lina He³, Yiu Sing Tsang33, Ying-San Di4, Sheng-Tao Liang4, \\ Qing $X_{i a}{ }^{\boxplus}$ \\ 1. Department of Oncology, State Key Laboratory for Oncogenes and Related Genes, Renji Hospital, School of Medicine, Shanghai Jiaotong University, \\ Shanghai Cancer Institute, Shanghai, 200127, China. \\ 2. Department of Laboratory Medicine, The Third Affiliated Hospital of Sun Yat-sen University, Guangzhou, 510630, China. \\ 3. Department of Basic Medical Sciences, Shanghai Jiao Tong University School of Medicine, Shanghai, 200025, China. \\ 4. Department of Oncology, Baoshan Branch Hospital, Renji Hospital, School of Medicine, Shanghai Jiaotong University, Shanghai, 200436, China. \\ \#These authors contributed equally to this work.
}

$\square$ Corresponding author: Qing Xia, Department of Oncology, State Key Laboratory for Oncogenes and Related Genes, Renji Hospital, School of Medicine, Shanghai Jiaotong University, Shanghai Cancer Institute, 160 Pujian Road, Shanghai 200127, China. Tel: +86-21-68385559, E-mail: jingtianxq@163.com.

(c) The author(s). This is an open access article distributed under the terms of the Creative Commons Attribution License (https://creativecommons.org/licenses/by/4.0/). See http://ivyspring.com/terms for full terms and conditions.

Received: 2020.03.23; Accepted: 2020.05.22; Published: 2020.06.06

\begin{abstract}
Although elevated neutrophil-to-lymphocyte ratio (NLR) and platelet-to-lymphocyte ratio (PLR) have been reported to be inverse prognostic predictors of survival in patients with pancreatic cancer (PC), the comparison of their prognostic roles in patients with PC undergoing gemcitabine-based chemotherapy and 5-fluorouracil (5-FU) remains unclear. This study was designed and performed to determine the predictive roles of NLR and PLR in patients diagnosed with PC who underwent one of these two regimens. We retrospectively enrolled 95 patients diagnosed with $P C$ undergoing supportive care, gemcitabine-based chemotherapy or 5-FU therapy from January 2015 to October 2018. Univariate and multivariate Cox regression analyses were done to identify clinicopathological predictors of time to treatment failure (TTF) and overall survival (OS), including pretreatment NLR and PLR. The statistical data showed that pretreatment NLR was significantly associated with metastasis. Among all analyzed variables, pretreatment NLR was an independent prognostic predictor of both TTF and OS of patients with PC, with NLR>4.0 predicting worse survival. PLR, however, didn't independently predict TTF or OS. There were no significant difference in the OS of patients undergoing gemcitabine-based regimens and 5-FU regimens when divided into two subgroups: NLR $\leq 4.0$ and $>4.0$. In conclusion, pretreatment NLR is a promising independent outcome predictor for patients with PC, while NLR might not be a suitable factor in the selection of regimens for patients with PC.
\end{abstract}

Key words: neutrophil-to-lymphocyte ratio; platelet-to-lymphocyte ratio; pancreatic cancer; prognosis; gemcitabine; 5-fluorouracil

\section{Introduction}

Pancreatic cancer (PC) is one of the most lethal cancers that remains a challenging medical problem for many years. The most common form is pancreatic ductal adenocarcinoma (PDA), the tenth most common solid cancer and the fourth leading cause of death from cancer in the United States [1]. The prognosis of PC is very poor, with the 5-year survival rate of only $7 \%$ [2]. Unfortunately, there are no specific symptoms. Patients at early stage are usually symptom-free, and PC in those who present with unspecific abdominal discomfort, weight loss or more specific jaundice are already in advanced stage. Most patients are diagnosed with metastatic disease and 
few show a sustained response to chemo- or radiation therapy [3].

Considering the poor prognostic outcome of patients with PC, efforts have been made to explore the predictive factors of this malignancy. It has been recognized that inflammatory responses play decisive roles at different stages of tumor development [4], and several inflammatory biomarkers have been proposed for the evaluation of cancer patients. Recently, pretreatment neutrophil-to-lymphocyte ratio (NLR) has been revealed to be a promising prognostic predictor for pancreatic cancer by several studies, where low NLR stands for better survival in patients with pancreatic cancer $[5,6]$. NLR is calculated by neutrophil count divided by lymphocyte count, which can be easily obtained by routine blood tests. Previous studies indicated that a high level of NLR might be significantly associated with poorer prognosis of several tumors, including colorectal cancer, lung cancer, gastric cancer, esophageal cancer and so on [7-10]. There are also evidences suggesting that platelet-to-lymphocyte (PLR), as another easily accessible biomarker that is calculated by platelet count divided by lymphocyte count, may also be a prognostic predictor of a variety of malignancies such as ovarian cancer [11], breast cancer [12] and nonsmall-cell lung cancer [13], where low PLR suggests a better outcome.

This study was designed and performed to determine the association between both pretreatment NLR and PLR and survival of patients with PC treated with supportive care, gemcitabine-based chemotherapy or 5-FU therapy.

\section{Materials and Methods}

\section{Patients and treatment}

Searching the database system of electronic medical charts, we collected clinical data of patients with the diagnosis of pancreatic cancer referred to Renji Hospital affiliated to Shanghai Jiao Tong University School of Medicine between January 2015 and October 2018. Patients whose diagnosis was PC were included in the study. Patients without available data or those with infection, regimens with steroids or aspirin, autoimmune disease or other conditions that might possibly confounded neutrophil count, lymphocyte count or platelet were excluded. In this study, patients underwent supportive care, gemcitabine-based therapy or 5-FU therapy. Gemcitabine-based therapy included gemcitabine monotherapy and gemcitabine combination therapy. Treatment was not terminated until tumor recurrence, tumor progression, treatment toxicity or patients' requirement for withdrawal. The doses and regimens were adjusted by corresponding physicians in accordance with adverse events and general conditions of the individual patient.

\section{Clinical and laboratory data collection}

Patients included were either chemotherapynaïve or chemotherapy-free for at least one month before they were referred to Renji Hospital affiliated to Shanghai Jiao Tong University. Baseline data was collected before treatment start. The history of patients was taken on the first days of hospitalization. Karnofsky performance status (KPS) was evaluated by treating physicians before the commencement of chemotherapy. Tumor location and TNM (tumor, lymph node and metastasis) staging were judged from the results of computed tomography (CT) or positron emission tomography (PET) in accordance with the $7^{\text {th }}$ edition of the AJCC Cancer Staging Manual. Biological markers, including carbohydrate antigen 19-9 (CA 19-9), carcinoembryonic antigen (CEA), carbohydrate antigen-125 (CA-125), neutrophil count, lymphocyte count and platelet count were collected within 3 weeks before the first cycle of chemotherapy as the pretreatment data. NLR was defined by the absolute neutrophil count divided by the absolute lymphocyte count. Likewise, PLR was defined by the absolute platelet count divided by the absolute lymphocyte count. All consecutive parameters were categorized for the further analysis as follows: age ( $\leq 65$ or $>65$ years), body mass index (BMI) $(\leq 18.5$, $18.5-24.0$ or $>24.0)$, CA $19-9(\leq 1000$ or $>1000 \mathrm{U} / \mathrm{ml})$, CEA $(\leq 5$ or $>5 \mathrm{ng} / \mathrm{ml})$, CA-125 $(\leq 38$ or $>38 \mathrm{U} / \mathrm{ml})$. Cut-off values were set on the basis of previous studies [14, 15].

\section{Statistical analysis}

The $\chi^{2}$ test or Fisher's exact test were used to compare baseline patient characteristics that were categorized variables. The association between pretreatment NLR and the time to treatment failure (TTF) as well as overall survival (OS) was evaluated, so was pretreatment PLR. TTF was defined as the time from the date of chemotherapy initiation to the date of termination due to various reasons, including tumor recurrence, tumor progression, treatment toxicity or patients' requirement for withdrawal. If a patient had not reached the endpoint caused by any of these reasons, TTF was censored at the time of the last follow-up. Recurrence and progression were determined using CT or PET. OS was calculated from the date of chemotherapy initiation to the date of death for any reason, or censored at the date of the last follow-up if the endpoint event was not observed. Univariate and multivariate Cox regression analyses were done to identify clinicopathological predictors of 
TTF and OS, including age, gender, BMI, KPS, personal history, diabetes at diagnosis, tumor location, TNM stage, CA 19-9, CEA, CA-125 and pretreatment NLR and PLR. The differences of TTF and OS were compared utilizing the Kaplan-Meier method with log-rank tests for survival plot depiction and Cox-regression analysis for the evaluation of hazard ratio (HR) and its 95\% confidence interval (95\% CI).

In order to set the cut-off points of both NLR and PLR, Receiver Operating Curves (ROC) were depicted. The classification variable was long vs short-term survival ( $>6$ vs $\leq 6$ months). For one thing, the choice of the 6 months as the division point was due to the convenience of the study, since a relevant proportion of patients $(47.4 \%)$ were classified as short-term survivors and only 4 patients had a follow-up less than 6 months. For another, the majority of patients included in this study were in stage IV, whose median survival was only 4-6 months [16], so the choice of the 6 months was feasible. The effects of potential prognostic predictors were tested by Cox regression. Only variables with a statistical significance in univariate analysis were investigated in multivariate analysis.

The statistical significance of all tests was twosided, $p<0.05$. Analyses and calculation were done by IBM SPSS Statistics 24.0.

\section{Regulatory consideration}

This study is approved by the Ethic Committee of Renji Hospital affiliated to Shanghai Jiao Tong University as stipulated by the Declaration of Helsinki.

\section{Results}

A total of 103 patients diagnosed with pancreatic cancer were referred to Renji Hospital affiliated to Shanghai Jiao Tong University School of Medicine between January 2015 and October 2018. All patients were histologically confirmed to have PC by biopsy, from which 4 patients with unavailable data and 4 patients who had infections, steroid regimens or autoimmune disease at the time of baseline data collection were excluded. Finnally, 95 patients were enrolled in the study.

The demographics and characteristics of all patients were shown in Table 1. Among the 95 patients in the study, 56 were male and 39 were female. The median age at baseline was 62 years (range, 42-83). BMI ranged from 14.4 to $28.1 \mathrm{~kg} / \mathrm{m}^{2}$, with the median of $20.8 \mathrm{~kg} / \mathrm{m}^{2}$ and $42.1 \%$ patients had $\mathrm{KPS} \leq 80$. As for the investigation of risk factors of PC, $32.6 \%$ of patients had a history of smoking while $16.8 \%$ had a history of alcohol intake. Moreover,
$13.7 \%$ of patients had a family history of cancer and $32.6 \%$ patients had diabetes at the time of PC diagnosis. Histologically, $87.4 \%$ patients were confirmed to have PC while others had neuroendocrine tumor and mucinous carcinoma. Tumor in $42.1 \%$ patients located in the head of pancreas and distantly metastasized in $72.6 \%$ patients. Routine biochemical tests performed before the commencement of treatment were collected as baseline data, some of which were long recognized prognostic predictors. In terms of therapy, $12.6 \%$ patients underwent supportive care, whereas 53.7\% patients were treated by gemcitabine-based therapy and $33.7 \%$ patients by 5 -FU. The median neutrophil count was $3.60^{*} 10^{9} / \mathrm{L}$, with the range of 1.44 $15.75^{\star} 10^{9} / \mathrm{L}$. The median lymphocyte count was $1.42 * 10^{9} / \mathrm{L}$, ranging from $0.50^{*} 10^{9}$ to $4.50^{*} 10^{9} / \mathrm{L}$. Median NLR was 2.6 (range, 0.5-18.0) with 27 (28.4\%) patients having NLR>4.0 and that of PLR was 148 (range, 51-785) with 39 (41.1\%) patients having PLR>169. Among all 95 patients, 39 (41.1\%) presented with the biomarker CA 19-9>1000 U/ml, 47 (49.5\%) with CEA $>5 \mathrm{ng} / \mathrm{ml}$ and 49 (51.6\%) with CA-125>38 $\mathrm{U} / \mathrm{ml}$.

Until October 2018, 72 patients reached TTF endpoint and 45 patients reached OS endpoint. For better utility of NLR as a predictor for the prognosis of patients with PC, a ROC curve was constructed to find the cut-off point of NLR. The area under curve (AUC) was 0.754 . A fixed cut-off value of 4.0 was taken for the analysis, yielding a sensitivity of $80.0 \%$ and a specificity of $60.0 \%$ (Figure 1A). With NLR=4.0 as the cut-off point, it was confirmed that pretreatment NLR was significantly associated with TTF, with median TTF of 8.0 months and 2.0 months for patients with NLR $\leq 4.0$ and $>4.0$ respectively, $\mathrm{HR}=3.158$ (95\% CI, 1.805-5.527), $p=0.0001$ (Figure 2A). NLR was also significantly associated with OS. The median OS of patients with NLR $\leq 4.0$ was 21.0 months and that of patients with NLR>4.0 was 5.0 months, $\mathrm{HR}=4.090(\mathrm{HR}=2.073-8.071), p=0.0001$ (Figure 2B). A ROC curve was also constructed to find the appropriate cut-off point of PLR. The AUC was 0.645. PLR=169 was found to be the best cut-off point in this study, with a sensitivity of $53.3 \%$ and a specificity of $70.0 \%$ (Figure 1B). Median TTF of PLR $\leq 169$ and $>169$ was 6.0 months and 3.0 months respectively, $\mathrm{HR}=1.511$ (95\% CI, 0.940-2.431), $p=0.088$ (Figure 2C). And median OS of PLR $\leq 169$ and $>169$ was 17.0 months and 10.0 months respectively, HR=1.683 (95\% CI, 0.917-3.089), $p=0.089$ (Figure 2D). The associations between NLR subgroups and biomarker CA 19-9 $(p=0.672)$, CEA $(p=0.455)$ and CA-125 $(p=0.625)$ were found not of significance (Table 1 ). 
Table 1. Characteristics of the 95 patients in study

\begin{tabular}{|c|c|c|c|c|}
\hline Characteristics & Category $(n=95)$ & Pretreatment NLR $\leq 4.0(\mathrm{n}=68)$ & Pretreatment NLR >4.0 $(\mathrm{n}=27)$ & $p$ value \\
\hline \multicolumn{5}{|l|}{ Gender } \\
\hline Male & $56(58.9)$ & 39(57.4) & $17(63.0)$ & \\
\hline Female & $39(41.1)$ & $29(42.6)$ & 10(37.0) & 0.586 \\
\hline \multicolumn{5}{|l|}{ Age } \\
\hline$\leq 65$ years & $66(69.5)$ & $46(67.6)$ & 20(74.1) & \\
\hline$>65$ years & 29(30.5) & $22(32.4)$ & $7(25.9)$ & 0.540 \\
\hline \multicolumn{5}{|l|}{ BMI } \\
\hline $18.5-24.0$ & $66(69.5)$ & $48(70.6)$ & $18(66.7)$ & \\
\hline$\leq 18.5$ & $12(12.6)$ & $9(13.2)$ & $3(11.1)$ & \\
\hline$>24.0$ & $17(17.9)$ & $11(16.2)$ & $6(22.2)$ & 0.776 \\
\hline \multicolumn{5}{|l|}{ KPS } \\
\hline$\leq 80$ & $40(42.1)$ & $26(38.2)$ & $14(51.9)$ & \\
\hline$>80$ & $55(57.9)$ & $42(61.8)$ & 13(48.1) & 0.225 \\
\hline \multicolumn{5}{|l|}{ History of Smoking } \\
\hline Yes & $31(32.6)$ & 19(27.9) & $12(44.4)$ & \\
\hline No & 64(67.4) & $49(72.1)$ & $15(55.6)$ & 0.122 \\
\hline \multicolumn{5}{|c|}{ History of Alcohol Intake } \\
\hline Yes & $16(16.8)$ & $8(11.8)$ & $8(29.6)$ & \\
\hline No & $79(83.2)$ & $60(88.2)$ & 19(70.4) & 0.036 \\
\hline \multicolumn{5}{|c|}{ Family History of Cancer } \\
\hline Yes & $13(13.7)$ & $9(13.2)$ & $4(14.8)$ & \\
\hline No & $82(86.3)$ & $59(86.8)$ & $23(85.2)$ & 0.840 \\
\hline \multicolumn{5}{|c|}{ Diabetes at Diagnosis } \\
\hline Yes & $31(32.6)$ & $23(33.8)$ & $8(29.6)$ & \\
\hline No & $64(67.4)$ & $45(66.2)$ & 19(70.4) & 0.694 \\
\hline \multicolumn{5}{|l|}{ Histology } \\
\hline PDA & $83(87.4)$ & $61(89.7)$ & $22(81.5)$ & \\
\hline Others & $12(12.6)$ & $7(10.3)$ & $5(18.5)$ & 0.276 \\
\hline \multicolumn{5}{|l|}{ T Stage } \\
\hline $\mathrm{T} 1$ & $8(8.4)$ & $7(10.3)$ & $1(3.7)$ & \\
\hline T2 & $31(32.6)$ & $20(29.4)$ & $11(40.7)$ & \\
\hline T3 & $28(29.5)$ & $20(29.4)$ & $8(29.6)$ & \\
\hline T4 & $28(29.5)$ & $21(30.9)$ & $7(25.9)$ & 0.590 \\
\hline \multicolumn{5}{|l|}{ N Stage } \\
\hline No & $25(26.3)$ & $23(33.8)$ & $2(7.4)$ & \\
\hline N1 & $70(73.7)$ & $45(66.2)$ & 25(92.6) & 0.008 \\
\hline \multicolumn{5}{|l|}{ M Stage } \\
\hline M0 & $26(27.4)$ & $25(36.8)$ & $1(3.7)$ & \\
\hline M1 & $69(72.6)$ & $43(63.2)$ & $26(96.3)$ & 0.001 \\
\hline \multicolumn{5}{|l|}{ Metastatic Site(s) } \\
\hline 0 & $26(27.4)$ & $25(36.8)$ & $1(3.7)$ & \\
\hline 1 & $37(38.9)$ & $26(38.2)$ & $11(40.7)$ & \\
\hline More than 1 & $32(33.7)$ & $17(25.0)$ & 15(55.6) & 0.001 \\
\hline \multicolumn{5}{|l|}{ Tumor Location } \\
\hline Head & $40(42.1)$ & $29(42.6)$ & $11(40.7)$ & \\
\hline Others & $55(57.9)$ & 39(57.4) & $16(59.3)$ & 0.865 \\
\hline \multicolumn{5}{|l|}{ CA 19-9 } \\
\hline$\leq 1000 \mathrm{U} / \mathrm{ml}$ & $56(58.9)$ & $41(60.3)$ & 15(55.6) & \\
\hline$>1000 \mathrm{U} / \mathrm{ml}$ & 39(41.1) & $27(39.7)$ & $12(44.4)$ & 0.672 \\
\hline \multicolumn{5}{|l|}{ CA-125 } \\
\hline$\leq 38 \mathrm{U} / \mathrm{ml}$ & $39(41.1)$ & $34(50.0)$ & $12(44.4)$ & \\
\hline$>38 \mathrm{U} / \mathrm{ml}$ & $56(58.9)$ & $34(50.0)$ & 15(55.6) & 0.625 \\
\hline \multicolumn{5}{|l|}{ CEA } \\
\hline$\leq 5 \mathrm{ng} / \mathrm{ml}$ & $48(50.5)$ & $36(52.9)$ & $12(44.4)$ & \\
\hline$>5 \mathrm{ng} / \mathrm{ml}$ & $47(49.5)$ & 32(47.1) & $15(55.6)$ & 0.455 \\
\hline Therapy & & & & \\
\hline Supportive care & $12(12.6)$ & $6(8.8)$ & $6(22.2)$ & \\
\hline Gemcitabine-based & $51(53.7)$ & 39(57.4) & $12(44.4)$ & \\
\hline 5-FU & $32(33.7)$ & $23(33.8)$ & $9(33.3)$ & 0.187 \\
\hline
\end{tabular}
19-9: carbohydrate antigen 19-9, CEA: carcinoembryonic antigen, CA-125: carbohydrate antigen-125, NLR: neutrophil-to-lymphocyte ratio

It was uncovered that NLR was significantly associated with $M$ stage, where NLR $>4.0$ suggested a more likely metastatic scenario $(p=0.001)$. And in those patients with localized tumors at the time of diagnosis, their NLR was almost all below $4.0(25 / 26)$. Such an association might partially explain the 
association between NLR and prognosis for patients with metastatic PC who undoubtedly tend to have a worse prognosis. Nevertheless, PLR was not found to be significantly associated with tumor metastasis $(p=0.211)$.

To evaluate whether NLR and PLR were independent predictors of prognosis, univariate and multivariate Cox-regression analyses for both TTF and OS were performed (Table 2). In the assessment of potential prognostic variables of TTF, N stage, $\mathrm{M}$ stage, CA 19-9, CA-125, CEA, pretreatment NLR were found to be significantly associated with TTF at univariate Cox-regression analysis. At multivariate analysis, only NLR had statistically significant association with TTF, the HR of NLR being 3.158 (95\% CI, 1.805-5.527), $p=0.0001$, although $\mathrm{M}$ stage exhibited a marginal significance $(p=0.051)$. At univariate analysis of OS, it was found that $\mathrm{N}$ stage, $\mathrm{M}$ stage, $\mathrm{CA}$ 19-9, CA-125, CEA, pretreatment NLR were significantly associated with OS. And NLR was proved to be an independent prognostic factor of OS, HR being 4.090 (95\% CI 2.073-8.071). $p=0.0001$. Meanwhile $\mathrm{N}$ stage also presented a statistical significance $(p=0.012)$. In univariable analysis, pretreatment PLR didn't show any significant association with TTF (HR=1.511[0.940-2.431], $p=0.088$, Figure 2C) or OS (HR=1.683[0.917-3.089], $p=0.089$, Figure 2D).

Among the 83 patients selected for the study that underwent chemotherapy, the regimens of 51 patients were gemcitabine-based and that of the others received 5-FU containing regimens. Gemcitabine-

A

NLR

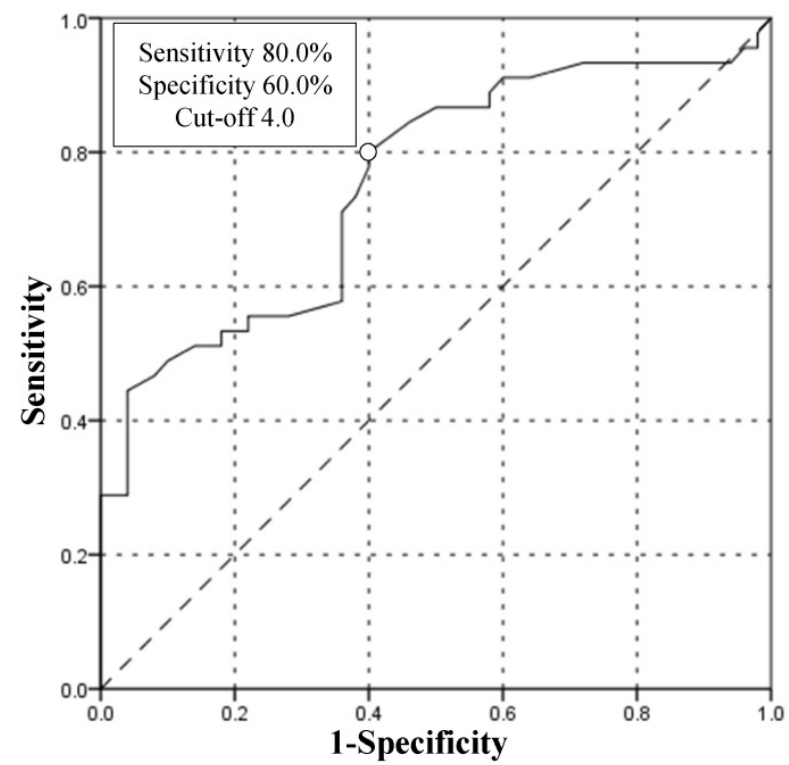

based therapy displayed a minor superior OS when compared to 5-FU, with median OS of 17.0 months and 15.0 months respectively ( $\mathrm{HR}=1.525[0.776-2.995]$ ). However, this was not of statistical significance $(p=0.217$, Figure 3). To evaluate the influence of NLR on the type of chemotherapy, the association between chemotherapy and OS was analyzed by separating patients into low and high pretreatment NLR. No significant interaction was found, with no difference between gemcitabine-based and 5-FU in the low NLR subgroup $(p=0.310)$ and the high NLR subgroup $(p=0.409)$.

\section{Discussion}

NLR has been confirmed to be a prognostic predictor in various cancers that significantly correlates with response rates, therapeutic effects and survival rates [7-10]. For patients with pancreatic cancer, high pretreatment NLR is found to be an unfavorable predictor of OS and TTF, and some studies pointed out that patients with high pretreatment NLR would tend to have a longer OS or PFS, if their NLR was lowered after the initiation of chemotherapy $[17,18]$. In the present study, we demonstrated that pretreatment NLR independently predicted the prognosis of patients with advanced or localized PC, even after adjusting for potential confounding factors. But PLR failed to independently predict the prognostic outcome in terms of both TTF and OS.

B

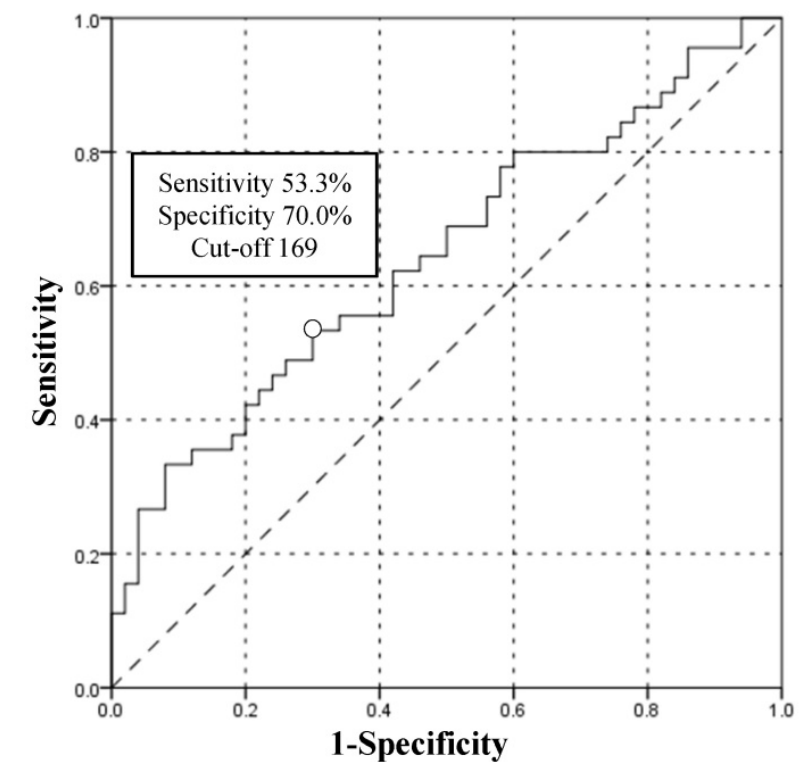

Figure 1. Receiver Operating Curve (ROC) analysis constructed to find the best cut-off point of neutrophil-to-lymphocyte ratio (NLR) and platelet-to-lymphocyte ratio (PLR). (A) ROC analysis of NLR. (B) ROC analysis of PLR. 
A

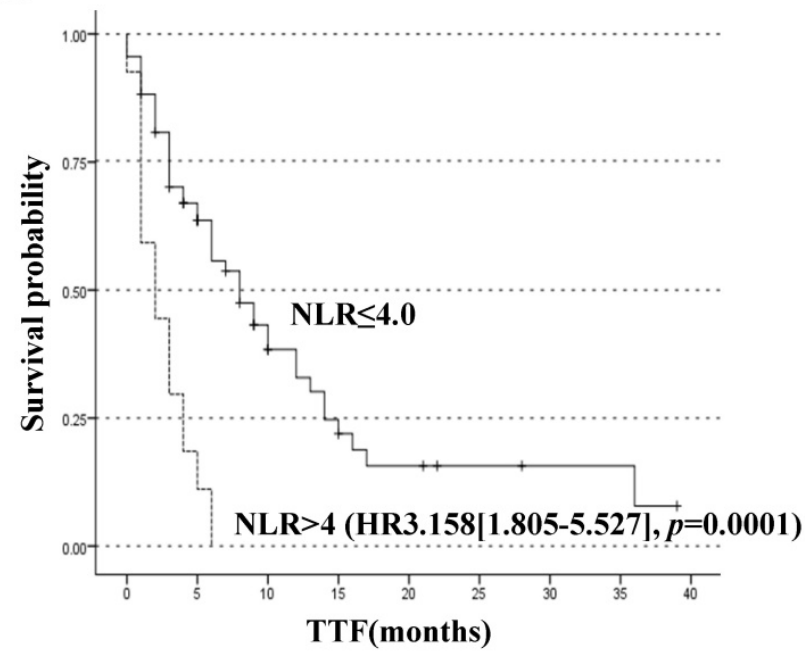

C

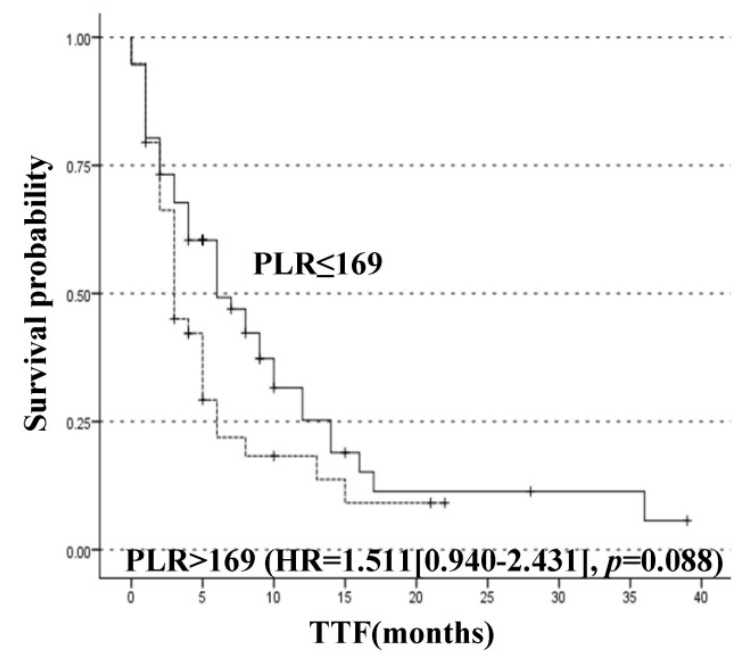

B

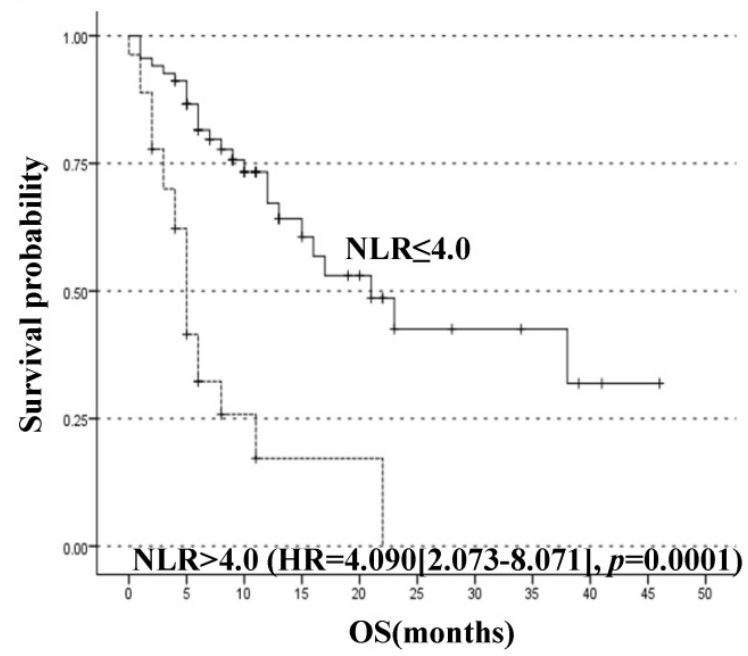

D

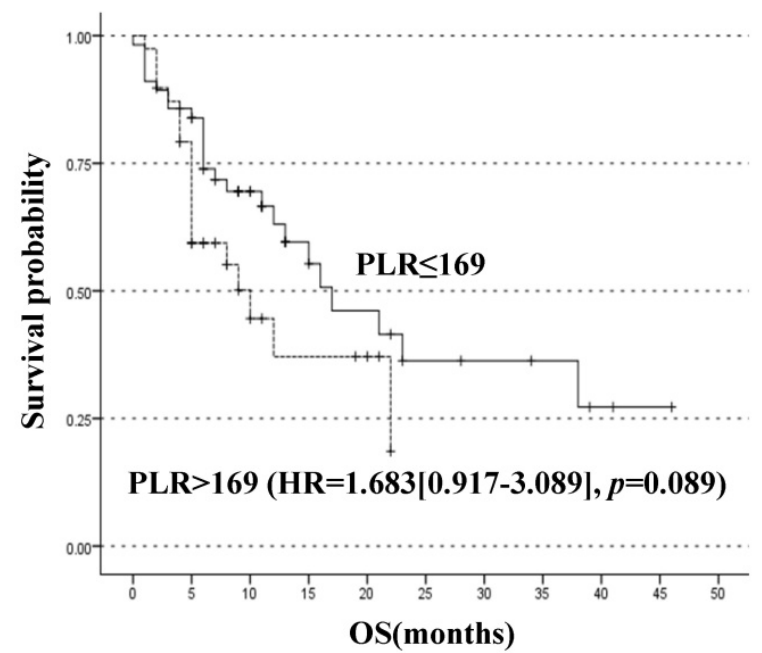

Figure 2. Kaplan-Meier curves stratified by the pretreatment levels of NLR and PLR. (A) Time to treatment failure (TTF) and (B) Overall survival (OS) of patients classified by NLR $\leq 4.0$ or $>4.0$. (C)TTF and (D) OS of patients classified by PLR $\leq 169$ or $>169$.

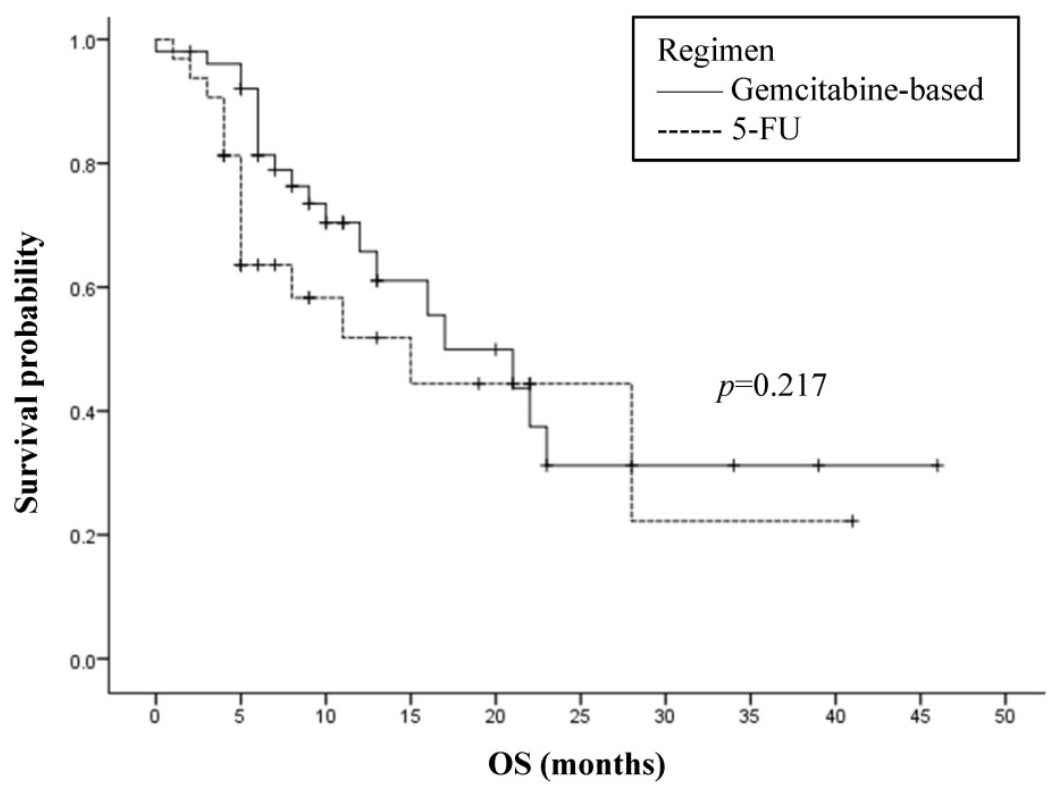

Figure 3. Overall survival (OS) and first-line chemotherapy regimen in 83 analyzed patients. 5-FU: 5-fluorouracil. 
Table 2. Univariate and multivariate Cox-regression analysis for time to treatment failure and overall survival

\begin{tabular}{|c|c|c|c|c|c|}
\hline \multirow{2}{*}{$\begin{array}{l}\text { Factor } \\
\text { ( } \mathrm{n}=\text { number of patients with data } \\
\text { available) }\end{array}$} & \multirow[t]{2}{*}{ n. (\%) } & \multicolumn{2}{|l|}{ Univariate analysis } & \multicolumn{2}{|l|}{ Multivariate analysis } \\
\hline & & TTF & OS & TTF & OS \\
\hline & & HR $(95 \% \mathrm{CI}), p$ & HR $(95 \% \mathrm{CI}), p$ & $\mathrm{HR}(95 \% \mathrm{CI}), p$ & $\mathrm{HR}(95 \% \mathrm{CI}), p$ \\
\hline \multicolumn{6}{|l|}{ Age $(n=95)$} \\
\hline$\leq 65$ years & $66(69.5)$ & 1 & 1 & & \\
\hline$>65$ years & $29(30.5)$ & $1.359(0.813-2.272), 0.240$ & $0.934(0.500-1.742), 0.829$ & & \\
\hline \multicolumn{6}{|l|}{ Gender $(n=95)$} \\
\hline Male & $56(58.9)$ & 1 & 1 & & \\
\hline Female & $39(41.1)$ & $0.754(0.465-1.223), 0.251$ & $0.666(0.357-1.249), 0.203$ & & \\
\hline \multicolumn{6}{|l|}{ BMI (n=95) } \\
\hline $18.5-24 \mathrm{~kg} / \mathrm{m}^{2}$ & $66(69.5)$ & 1 & 1 & & \\
\hline$\leq 18.5 \mathrm{~kg} / \mathrm{m}^{2}$ & 17(17.9) & 1.289(0.683-2.433), & $1.958(0.944-4.062)$ & & \\
\hline$>24 \mathrm{~kg} / \mathrm{m}^{2}$ & $12(12.6)$ & $0.672(0.327-1.382), 0.351$ & $0.694(0.268-1.799), 0.100$ & & \\
\hline \multicolumn{6}{|l|}{ KPS $(n=95)$} \\
\hline$\leq 80$ & $40(42.1)$ & 1 & 1 & & \\
\hline$>80$ & $55(57.9)$ & $0.937(0.584-1.502), 0.787$ & $0.837(0.461-1.520), 0.558$ & & \\
\hline \multicolumn{6}{|l|}{ Smoking ( $n=95)$} \\
\hline No & $64(67.4)$ & 1 & 1 & & \\
\hline Yes & $31(32.6)$ & $1.330(0.815-2.171), 0.254$ & $1.188(0.632-2.231), 0.593$ & & \\
\hline \multicolumn{6}{|l|}{ Alcohol intake $(n=95)$} \\
\hline No & $79(83.2)$ & 1 & 1 & & \\
\hline Yes & $16(16.8)$ & $1.462(0.811-2.638), 0.207$ & $1.564(0.767-3.186), 0.215$ & & \\
\hline \multicolumn{6}{|l|}{ Family history of Cancer $(n=95)$} \\
\hline No & $64(67.4)$ & 1 & 1 & & \\
\hline Yes & $31(32.6)$ & $1.028(0.537-1.968), 0.934$ & $0.694(0.273-1.762), 0.440$ & & \\
\hline \multicolumn{6}{|l|}{ Diabetes at diagnosis $(n=95)$} \\
\hline No & $40(42.1)$ & 1 & 1 & & \\
\hline Yes & $55(57.9)$ & $1.068(0.649-1.759), 0.796$ & $1.022(0.549-1.904), 0.945$ & & \\
\hline \multicolumn{6}{|l|}{ Tumor Location $(n=95)$} \\
\hline Head & $40(42.1)$ & 1 & 1 & & \\
\hline Others & $55(57.9)$ & $0.860(0.533-1.389), 0.538$ & $0.834(0.458-1.518), 0.551$ & & \\
\hline \multicolumn{6}{|l|}{ T Stage $(n=95)$} \\
\hline $\mathrm{T} 1, \mathrm{~T} 2$ & $42(44.2)$ & 1 & 1 & & \\
\hline $\mathrm{T} 3, \mathrm{~T} 4$ & $53(55.8)$ & $1.430(0.891-2.296), 0.137$ & $1.215(0.670-2.202), 0.522$ & & \\
\hline \multicolumn{6}{|l|}{$N$ stage $(n=95)$} \\
\hline No & $25(26.3)$ & 1 & 1 & 1 & 1 \\
\hline N1 & $70(73.7)$ & $2.271(1.276-4.043), 0.005$ & $3.225(1.419-7.326), 0.005$ & $1.422(0.757-2.671), 0.273$ & $3.180(1.288-7.850), 0.012$ \\
\hline \multicolumn{6}{|l|}{ M Stage $(n=95)$} \\
\hline M0 & $26(27.4)$ & 1 & 1 & 1 & 1 \\
\hline M1 & $69(72.6)$ & $3.715(1.981-6.968), 0.0001$ & $5.359(2.067-13.893), 0.001$ & $2.148(0.997-4.626), 0.051$ & $1.306(0.410-4.160), 0.651$ \\
\hline Histology $(n=95)$ & & & & & \\
\hline PDA & $83(87.4)$ & 1 & 1 & & \\
\hline Others & $12(12.6)$ & $1.417(0.741-2.710), 0.292$ & $1.452(0.643-3.281), 0.369$ & & \\
\hline CA 19-9 $(n=95)$ & & & & & \\
\hline$\leq 1000 \mathrm{U} / \mathrm{ml}$ & $56(58.9)$ & 1 & 1 & 1 & 1 \\
\hline$>1000 \mathrm{U} / \mathrm{ml}$ & $39(41.1)$ & $1.868(1.142-3.054), 0.013$ & $2.987(1.608-5.550), 0.001$ & $1.434(0.815-2.526), 0.212$ & $1.981(0.953-4.117), 0.067$ \\
\hline CA-125 (n=95) & & & & & \\
\hline$\leq 38 \mathrm{U} / \mathrm{ml}$ & $46(48.4)$ & 1 & 1 & 1 & 1 \\
\hline$>38 \mathrm{U} / \mathrm{ml}$ & $49(51.6)$ & $1.999(1.230-3.249), 0.005$ & $2.209(1.176-4.150), 0.014$ & $1.393(0.832-2.333), 0.207$ & $1.382(0.710-2.688), 0.341$ \\
\hline CEA $(n=95)$ & & & & & \\
\hline$\leq 5 \mathrm{ng} / \mathrm{ml}$ & $48(50.5)$ & 1 & 1 & 1 & 1 \\
\hline$>5 \mathrm{ng} / \mathrm{ml}$ & $47(49.5)$ & $1.714(1.062-2.765), 0.027$ & $3.112(1.639-5.906), 0.001$ & $1.021(0.583-1.786), 0.942$ & $1.716(0.790-3.726), 0.173$ \\
\hline Pretreatment NLR $(n=95)$ & & & & & \\
\hline$\leq 4.0$ & $68(71.6)$ & 1 & 1 & 1 & 1 \\
\hline$>4.0$ & $27(28.4)$ & $4.067(2.362-7.002), 0.0001$ & $4.572(2.434-8.589), 0.0001$ & $3.158(1.805-5.527), 0.0001$ & $4.090(2.073-8.071), 0.0001$ \\
\hline Pretreatment PLR $(n=95)$ & & & & & \\
\hline$\leq 169$ & $56(58.9)$ & 1 & 1 & & \\
\hline$>169$ & $39(41.1)$ & $1.511(0.940-2.431), 0.088$ & $1.683(0.917-3.089), 0.089$ & & \\
\hline Type of therapy $(n=83)$ & & & & & \\
\hline Gemcitabine-based & $51(61.4)$ & 1 & 1 & & \\
\hline 5-FU & $32(38.5)$ & $1.141(0.683-1.908), 0.614$ & $1.525(0.776-2.995), 0.217$ & & \\
\hline
\end{tabular}

A number of studies have suggested the inverse association between both NLR and PLR and prognosis of patients with PC $[6,19]$. Although it has long been established that NLR and PLR both play roles in predicting the prognosis of various malignancies, the question which one is more predictive and suggestive is still debatable. The comparison between NLR and PLR was done to 
analyze which factor does better in predicting the prognosis of patients with PC.

A dynamic and mutualistic interaction between tumour cells and the surrounding stromal cells promotes the initiation, progression, metastasis and chemoresistance of solid tumours [20]. As important components of stromal cells, neutrophils are enriched in many types of cancers and high levels of neutrophils are closely associated with disease progression and poor clinical outcome [21]. Neutrophilia is a common phenomenon in PC. Multiple mechanisms have been hypothesized or demonstrated. It was proposed that neutrophil might aid metastasis of pancreatic cancer because it is able to mediate epithelial-mesenchymal transition (EMT) of cancer cells by secreting elastase [22]. Neutrophil can secrete vascular endothelial growth factor (VEGF) and matrix metalloproteinase 9 (MMP-9), which promotes angiogenesis thus facilitating the growth and metastases of PC [23]. Another study found that neutrophils can also promote tumor growth through converting senescent cancer cells into proliferating cancer cells via IL-1 receptor antagonist [24]. Besides, neutrophil may help with the creation of immunosuppressive microenvironment of pancreatic cancer by suppressing $\mathrm{CD}^{+} \mathrm{T}$ cells [25]. Pancreatic cancer is also associated with declined lymphocyte count, which may impair immune surveillance and defense. The proliferation of Lymphocytes is suppressed by various immunosuppressive cytokines during tumorigenesis, principally including transforming growth factor $\beta$ (TGF- $\beta$ ) and interleukin 10 (IL-10) [26]. The association between thrombocytosis and PC is still not well understood. Platelets can secrete tumor growth factors, such as VEGF, TGF- $\beta$, platelet-derived growth factor (PDGF), and insulin-like growth factor-1 (IGF1), which play critical roles in cancer angiogenesis and metastasis [27].

In this study, NLR did provide independent prognostic information of survival in terms of both TTF and OS, whereas PLR was not significantly associated with TTF or OS. Several studies have compared the predictive value of NLR and PLR in terms of survival, response rates to treatment and recurrence after resection. NLR was recognized as a promising prognostic predictor whereas the association of PLR with patient outcome seems to be controversial [28]. Hasegawa et al. reported that pretreatment NLR might be a useful predictive marker for the pathological response to preoperative therapy in pancreatic cancer patients, which pretreatment PLR failed to predict [29]. Martin et al. found both NLR and PLR were predictive of overall survival of patients with advanced $\mathrm{PC}$, despite a more powerful predictive value of NLR $(\mathrm{HR}=1.81$, $p=0.0007)$ than PLR $(\mathrm{HR}=1.64, p=0.007)$ [30]. Stotz et al. reported that NLR added independent prognostic information to other well established prognostic factors in patients with pancreatic cancer, regardless of the undergoing therapeutic modality $(\mathrm{HR}=2.532$, $p<0.001$ ), but PLR did not show any predictive value as for overall survival in patients with both inoperative $(p=0.612)$ and operative pancreatic cancer $(p=0.458)$ [31]. Yang et al. performed a meta-analysis and found that high peripheral blood PLR suggested a poor prognosis for patients with pancreatic cancer [32]. Kishi et al. analyzed 65 patients with pancreatic cancer and drew the conclusion that PLR was not associated with the prognosis of these patients [33]. As far as this study is concerned, PLR failed to show the same significant association as NLR did. NLR might possess a better predictive value than PLR does in patients with PC.

Early administration of chemotherapy is favoured for patients with advanced PC and the standard of chemotherapy has changed in the last few years in the treatment of PC [34]. 5-FU regimens were in common use prior to the adoption of gemcitabine as the chemotherapy for patients with PC. Nonetheless, according to several phase II and randomized controlled trials, both bolus and infusion leucovorin modulated 5-FU-containing regimens are associated with low response rates and survival inferior to that seen with gemcitabine [35, 36]. Still, 5 -FU remains an alternative for gemcitabineintolerant patients. In this study, when patients were subgrouped by the criterion of NLR $\leq 4.0$ and $>4.0$, the OS did not significantly vary between gemcitabinebased regimens and 5-FU regimens. NLR might not be a suitable factor in the selection of regimens for patients with PC.

We recognize several limitations in our study. Firstly, the research was retrospective with a relatively small sample size. Besides, the analysis of NLR and PLR for predictive values of different chemotherapeutic modalities was achieved by dividing the population into smaller chemotherapy subgroups. Moreover, the major drawback is that treatment assignment was not randomized. Finally, blood samples were not derived during follow-up, making it infeasible to analyze whether further change of NLR and PLR would predict prognosis more accurately.

In conclusion, pretreatment NLR is a promising independent outcome predictor for patients with pancreatic ductal adenocarcinoma. The predictive value of PLR might not be as good as NLR. NLR could be used to predict time to treatment failure and overall survival for these patients. 


\section{Acknowledgements}

This study was supported by Young Scientist Project of the National Natural Science Foundation of China (No. 81702843 and 81802403). The funder had no role in the study except for financial support.

\section{Authors' contributions}

Chen YG, Liao Y, Lam LK, He LN and Xia Q designed the idea and wrote the manuscript. Chen YG, Liao Y, Lam LK, He LN and Tsang YS collected the data and participated in the design of the study. All authors read and approved the manuscript.

\section{Competing Interests}

The authors have declared that no competing interest exists.

\section{References}

1. Siegel RL, Miller KD, Jemal A. Cancer Statistics, 2017. CA Cancer J Clin. 2017; 67: 7-30.

2. Miller KD, Siegel RL, Lin CC, Mariotto AB, Kramer JL, Rowland JH, et al. Cancer treatment and survivorship statistics, 2016. CA Cancer J Clin. 2016; 66: 271-89.

3. Yachida S, Jones S, Bozic I, Antal T, Leary R, Fu B, et al. Distant Metastasis Occurs Late during the Genetic Evolution of Pancreatic Cancer. Nature. 2010; 467(7319):1114-7.

4. Grivennikov SI, Greten FR, Karin M. Immunity, inflammation, and cancer. Cell. 2010; 140: 883-99.

5. Cheng H, Long F, Jaiswar M, Yang L, Wang C, Zhou Z. Prognostic role of the neutrophil-to-lymphocyte ratio in pancreatic cancer: a meta-analysis. Scientific Reports. 2015; 5: 11026

6. Zhou Y, Wei Q, Fan J, Cheng S, Ding W, Hua Z. Prognostic role of the neutrophil-to-lymphocyte ratio in pancreatic cancer: $\mathrm{A}$ meta-analysis containing 8252 patients. Clinica chimica acta; international journal of clinical chemistry. 2018; 479: 181.

7. Li M-X, Liu X-M, Zhang X-F, Zhang J-F, Wang W-L, Zhu Y, et al. Prognostic role of neutrophil-to-lymphocyte ratio in colorectal cancer: A systematic review and meta-analysis. International Journal of Cancer. 2014; 134: 2403-13.

8. Cedrés S, Martínez A, Martinez P, Navarro A, Zamora E, Mulet-Margalef N, et al. Neutrophil to lymphocyte ratio (NLR) as an indicator of poor prognosis in stage IV non-small cell lung cancer. Clinical and Translational Oncology. 2012; 14: 864-9.

9. Cai J, Yin J, Song JN. Study on significance of neutrophil to lymphocyte ratio(NLR) in patients with gastric cancer. Journal of Clinical \& Experimental Medicine. 2014.

10. Yang X, Huang Y, Feng JF, Liu JS. Prognostic significance of neutrophil-tolymphocyte ratio in esophageal cancer: a meta-analysis. Oncotargets \& Therapy. 2015; 2015: 789.

11. Supachai R, Siriwan T, Sumonmal M, Sunamchok S, Thaovalai T. Platelet to lymphocyte ratio as a prognostic factor for epithelial ovarian cancer. Journal of Gynecologic Oncology. 2012; 23: 265-73.

12. Krenn-Pilko S, Langsenlehner U, Thurner EM, Stojakovic T, Pichler M, Gerger A, et al. The elevated preoperative platelet-to-lymphocyte ratio predicts poor prognosis in breast cancer patients. British Journal of Cancer. 2014; 110: 2524.

13. Nikolić I, Kukulj S, Samaržija M, Jeleč V, Žarak M, Orehovec B, et al. Neutrophil-to-lymphocyte and platelet-to-lymphocyte ratio help identify patients with lung cancer, but do not differentiate between lung cancer subtypes. Croatian Medical Journal. 2016; 57: 287-92.

14. Xue P, Kanai M, Mori Y, Nishimura T, Uza N, Kodama Y, et al. Neutrophil-to-lymphocyte ratio for predicting palliative chemotherapy outcomes in advanced pancreatic cancer patients. Cancer Medicine. 2014; 3: 406-15

15. Krishnan S, Vishal Rana, Janjan NA, Abbruzzese JL, Mpas MSG, MPH PDMM, et al. Prognostic factors in patients with unresectable locally advanced pancreatic adenocarcinoma treated with chemoradiation. Cancer. 2010; 107: 2589-96.

16. Vincent A, Herman J, Schulick R, Hruban RH, Goggins M. Pancreatic cancer. The Lancet. 2011; 378: 607-20.

17. Formica V, Morelli C, Ferroni P, Nardecchia A, Tesauro M, Pellicori S, et al. Neutrophil/lymphocyte ratio helps select metastatic pancreatic cancer patients benefitting from oxaliplatin. Cancer Biomarkers. 2016; 17: 335.

18. Luo G, Guo M, Liu Z, Xiao Z, Jin K, Long J, et al. Blood NeutrophilLymphocyte Ratio Predicts Survival in Patients with Advanced Pancreatic
Cancer Treated with Chemotherapy. Annals of Surgical Oncology. 2015; 22: 670-6

19. Xu ZS, Zhang FP, Zhang Y, Ou-Yang YP, Yu XW, Wang WL, et al. Prognostic role of the pre-treatment platelet-lymphocyte ratio in pancreatic cancer: a meta-analysis. Oncotarget. 2017; 8: 99003-12.

20. Turley SJ, Cremasco V, Astarita JL. Immunological hallmarks of stromal cells in the tumour microenvironment. Nature reviews Immunology. 2015; 15: 669-82.

21. Ramachandran IR, Condamine T, Lin C, Herlihy SE, Garfall A, Vogl DT, et al. Bone marrow PMN-MDSCs and neutrophils are functionally similar in protection of multiple myeloma from chemotherapy. Cancer letters. 2016; 371: 117-24.

22. Gaida MM, Steffen TGe, Günther F, Tschaharganeh DF, Felix K, Bergmann F, et al. Polymorphonuclear neutrophils promote dyshesion of tumor cells and elastase-mediated degradation of E-cadherin in pancreatic tumors. European Journal of Immunology. 2012; 42: 3369-80.

23. Bausch D, Pausch T, Krauss T, Hopt UT, Fernandezdelcastillo C, Warshaw AL, et al. Neutrophil granulocyte derived MMP-9 is a VEGF independent functional component of the angiogenic switch in pancreatic ductal adenocarcinoma. Angiogenesis. 2011; 14: 235.

24. Di Mitri D, Toso A, Chen JJ, Sarti M, Pinton S, Jost TR, et al. Tumour-infiltrating Gr-1+ myeloid cells antagonize senescence in cancer. Nature. 2014; 515: 134-7.

25. Coffelt SB, Kersten K, Doornebal CW, Weiden J, Vrijland K, Hau C-S, et al. IL-17-producing $\gamma \delta \mathrm{T}$ cells and neutrophils conspire to promote breast cancer metastasis. Nature. 2015; 522: 345

26. Salazar-Onfray F, López MN, Mendoza-Naranjo A. Paradoxical effects of cytokines in tumor immune surveillance and tumor immune escape. Cytokine \& Growth Factor Reviews. 2007; 18: 171-82

27. Qi C, Li B, Guo S, Wei B, Shao C, Li J, et al. P-Selectin-Mediated Adhesion between Platelets and Tumor Cells Promotes Intestinal Tumorigenesis in ApcMin/+ Mice. International Journal of Biological Sciences. 2015; 11: 679-87.

28. Zhou Y, Cheng S, Fathy AH, Qian H, Zhao Y. Prognostic value of platelet-to-lymphocyte ratio in pancreatic cancer: a comprehensive meta-analysis of 17 cohort studies. Oncotargets \& Therapy. 2018; 11: 1899-908.

29. Hasegawa S, Eguchi H, Tomokuni A, Tomimaru Y, Asaoka T, Wada H, et al. Pre-treatment neutrophil to lymphocyte ratio as a predictive marker for pathological response to preoperative chemoradiotherapy in pancreatic cancer. Oncology Letters. 2016; 11: 1560-6.

30. Martin HL, Ohara K, Kiberu A, Van Hagen T, Davidson A, Khattak MA. Prognostic value of systemic inflammation-based markers in advanced pancreatic cancer. Intern Med J. 2014; 44: 676-82.

31. Stotz M, Gerger A, Eisner F, Szkandera J, Loibner H, Ress AL, et al. Increased neutrophil-lymphocyte ratio is a poor prognostic factor in patients with primary operable and inoperable pancreatic cancer. 2013; 109: 416-21.

32. Yang JJ, Hu ZG, Shi WX, Deng T, He SQ, Yuan SG. Prognostic significance of neutrophil to lymphocyte ratio in pancreatic cancer: A meta-analysis. World J Gastroenterol. 2015; 21: 2807-15.

33. Kishi T, Nakamura A, Itasaka S, Shibuya K, Matsumoto S, Kanai M, et al. Pretreatment C-reactive protein level predicts outcome and patterns of failure after chemoradiotherapy for locally advanced pancreatic cancer. Pancreatology. 2015; 15: 694-700.

34. Garrido-Laguna I, Hidalgo M. Pancreatic cancer: from state-of-the-art treatments to promising novel therapies. Nature reviews Clinical oncology. 2015; $12: 319-34$

35. Crown J, Casper ES, Botet J, Murray P, Kelsen DP. Lack of efficacy of high-dose leucovorin and fluorouracil in patients with advanced pancreatic adenocarcinoma. J Clin Oncol. 1991; 9: 1682-6.

36. Van Rijswijk RE, Jeziorski K, Wagener DJ, Van Laethem JL, Reuse S, Baron B, et al. Weekly high-dose 5-fluorouracil and folinic acid in metastatic pancreatic carcinoma: a phase II study of the EORTC GastroIntestinal Tract Cancer Cooperative Group. Eur J Cancer. 2004; 40: 2077-81. 